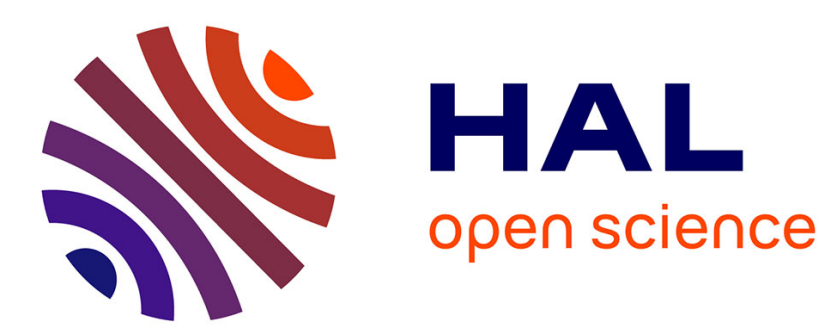

\title{
Diagnostics of noise acoustic sources
}

I. Fiks, N. Sidorovskaia, V. Turchin

\section{To cite this version:}

I. Fiks, N. Sidorovskaia, V. Turchin. Diagnostics of noise acoustic sources. Journal de Physique IV Proceedings, 1994, 04 (C5), pp.C7-1109-C7-1111. 10.1051/jp4:19945244 . jpa-00252932

\section{HAL Id: jpa-00252932 https://hal.science/jpa-00252932}

Submitted on 1 Jan 1994

HAL is a multi-disciplinary open access archive for the deposit and dissemination of scientific research documents, whether they are published or not. The documents may come from teaching and research institutions in France or abroad, or from public or private research centers.
L'archive ouverte pluridisciplinaire HAL, est destinée au dépôt et à la diffusion de documents scientifiques de niveau recherche, publiés ou non, émanant des établissements d'enseignement et de recherche français ou étrangers, des laboratoires publics ou privés. 


\title{
Diagnostics of noise acoustic sources
}

\author{
I.Sh. FIKS, N.A. SIDOROVSKAIA and V.I. TURCHIN \\ Institute of Applied Physics, Russian Academy of Sciences, 46 Uljanov St., 603600 Nizhny Novgorod, \\ Russia
}

\begin{abstract}
The diagnostics of the noise acoustical sources from near-field data is considered. The algorithms allowing the determination of the equivalent elementary source distribution along a radiator and the far-field reconstruction from near-field measurements are presented. Some components of the total reconstruction error are analyzed.
\end{abstract}

\section{INTRODUCTION}

At present the near-field (NF) methods are broadly used for the determination of the antenna radiation pattern. Since the 70 -s a reliable technology based on NF methods has been developed for the measurement of microwave antenna characteristics. Both the radiator far field and the amplitude and phase distribution of elementary sources on the radiator can be reconstructed with high accuracy by processing of the near-field data. In view of the fact that the measurements are made not far from a radiator, the main merits of $\mathrm{NF}$ methods are: the possibility of decreasing radiation power level and the reduction of error components caused by propagation medium and reverberation effects.

The development of NF methods in acoustics is their adoption for the characteristic measurements of extended sound sources with a priori unknown complex spectral power density (for example, for diagnostics of noise radiation of cars, ships and so on). The final aim here is the reconstruction of angular averaged intensity distribution in far field and the identification of equivalent distribution of acoustic elementary sources on the radiator from the measured data. In acoustic application NF methods should be developed in following directions. Firstly, reconstruction methods should be generalized for broad-band signals having random nature. In this case the second statistical moments of acoustical field depending on spatial coordinates and frequency should be estimated. Secondly, a robustness against noise should be investigated because in many cases actual acoustical signals do not exceed the background level. Thirdly, the radiator motion and signal propagation (at least, 
boundary reflections) should be taken into account.

\section{THE MAIN THEORETICAL CONCEPTS AND ALGORITHMS}

For low frequencies a most available measuring system is a linear array. On the other hand there is a broad enough class of acoustical radiators essentially oblong along one of coordinate axis (radiators with such geometry may be approximated by elementary source set on the segment of straight line). We have investigated that in this case the radiator characteristic reconstruction is carried out correctly. All mentioned-above conditions must be taken into account for the use of NF methods for the determination of acoustic radiator characteristics.

The theoretical part of NF method design consists in the design of algorithms of measured data transformation and in substantiation of their correction, whence the requirements on receiving system follow.

Proceeding from the random nature of radiation field, we propose the signal processing procedure should be divided into four main stages. The first stage is the received signal filtering by narrow bands. The second one is the algorithm of the spatial processing: the transformation of array signal vector $\vec{p}_{j}$ having passed through the narrow-band filter into a vector of momentary direction pattern $\hat{\vec{d}}_{j}$ or into a vector of momentary distribution of discrete equivalent sources on radiator $\widehat{\vec{m}}_{j}$ for every time point $j$ and for every band:

$$
\widehat{\vec{d}}_{j}=\Gamma_{j} \vec{p}_{j}
$$

During our research two basic algorithms of noise acoustic radiator characteristics reconstruction have been designed: the algorithm based on the high frequency approximation for solving appropriate integral equations (HFA-algorithm) and the algorithm of maximum likelihood estimation with regularization (MLER-algorithm).

The HFA-method had been originally developed for measurement of electromagnetic antenna characteristics, then we modified it for acoustic situation [1]. The HFA-algorithm is notable for simplicity of realization and physical interpretation. It permits to easily estimate the influence of measuring system parameters on final result. For the HFA-algorithm matrix $\Gamma$ doesn't depend on time point and is determined as:

$$
\Gamma_{k n}^{(H F A)}=d_{a} \sqrt{\frac{f}{c} \rho\left(x_{n}, \theta_{k}\right)} e^{-2 \pi i \frac{f}{c} l\left(x_{n}, \theta_{k}\right)},
$$

where $d_{a}$ - the sensor distance, $\mathrm{c}$ - the average sound velocity. Functions $\rho\left(x_{n}, \theta_{k}\right)$ and $l\left(x_{n}, \theta_{k}\right)$ is defined by only the angle $\theta_{k}$ and geometry of the mutual disposition of receiving array and the trajectory of the source motion.

But this algorithm is not optimum relative to noise and is basically orientated for the use in free space. These facts may lead to increase of the total error for measurements in inhomogeneous medium. The attempt of the design of optimum algorithm based on maximum likelihood principle was undertaken by us for overcoming these difficulties. Earlier use of maximum likelihood principle for estimation of acoustic radiator characteristics by NF measurements was considered [2,3] but only to non-random signals and high signal-noise ratio. For the MLER-algorithm realization we use statistics of second order obtained by linearization of maximum likelihood equations. For the MLER-algorithm the matrix $\Gamma_{j}$ is:

$$
\Gamma_{j}=U\left(G_{j}^{+} G_{j}+\varepsilon I\right)^{-1} G_{j}^{+},
$$


where the superscript + denotes conjugate transpose; $G_{j}$ is the matrix of mapping the equivalent sources into near-field samples; the matrix $U$ is the standard Fourier transformation of estimated $\widehat{\vec{m}}_{j}$ into the momentary direction pattern. The regularization procedure for $G_{j}^{+} G_{j}$ inversion indicates the presence of small eigen values in spectrum of this matrix. It means the impossibility of unbiased estimations of source characteristics by numerical methods. The optimum value of parameter $\varepsilon$ can be found from a priori known signal-noise ratio.

The third and fourth stages consist of the estimation of second order correlation (averaged intensity structure) by time averaging with weight coefficients and the compensation of external noise:

$$
D_{k k}=D\left(\theta_{k}\right)=\sum_{j} \gamma_{k, j}\left|\widehat{d}_{k, j}\right|^{2}-D_{k k}^{c o m p}
$$

The introduction of weight coefficients follows from the existence of angular sectors of trustworthy reconstruction (TRS) of direction pattern for current source location relative to array [1]. The estimation error is much smaller within the TRS than out of one. The $\gamma_{k, j}$ calculation for this procedure of the so-called "projection synthesis" is based on the determination of the TRS for model sources.

\section{RECONSTRUCTION ERROR}

Some components of total error of radiator characteristic reconstruction have been investigated. As the main components of total error the following factors have been chosen and analyzed:

1. the errors connected with different approximations for designing reconstruction algorithms (for example, approximate solution of integral equation, radiator identification by finite number of elementary sources, finite dimensions of receiving system);

2. the error caused by external noise;

3. the error connected with the non-identity of the receiving array transmission channels;

4. the error caused by the inaccurate estimation of the mutual disposition of radiator and receiving array elements;

5. the error connected with the inaccurate information about propagation channel and its fluctuations.

\section{OUTLOOK}

The results of an extensive simulation and the source characteristic reconstruction in natural experiments show that the estimates based on the above-mentioned algorithms have high accuracy. Furthermore, the MLER-algorithm allows to adapt the signal processing to complex source structure and to propagation medium by setting model Green function nearest the real one. It is believed that, besides being used for the investigation of acoustic object noise radiation, the MLER-algorithm can be developed for broad sphere of applied problems (for example, for diagnostics of hydrological and other ocean parameters; for acoustic monitoring in medicine and etc.).

\section{REFERENCES}

1. Turchin V.I., Fix I.Sh. et al., "Distant measurements of characteristics of moving radiators with complex spatial-time structure", Tomografic methods in physical-technical measurements, Moscow: VNIIFTRI 1990 pp. 159-180 (in Russian).

2. Borgiotti G.V. and Rosen E.M., J. Acoust. Soc. Am. 92(2), Pt. 1 (1992) 807-818

3. R. Stoughton, J. Acoust. Soc. Am. 92(2), Pt.1 (1992) 831-840 